\title{
Two Robust Homing Missile Guidance Laws Based on Sliding Mode Control Theory
}

\author{
K.R. Babu \\ I.G. Sarma \\ K.N.Swamy \\ Indian Institute of Science, Bangalore-560 012, India
}

\begin{abstract}
Two new guidance laws for short range homing missiles are developed by invoking the Sliding Mode Control (SMC) theory. Guidance law 1 is structured around the basic Proportional Navigation (PN), with an additive switching term, which is a function of the Line of Sight (LOS) rate alone. An adaptive procedure is suggested to select the gain of the switching term, in order to reduce chattering. This guidance law is nearly as simple to implement as the $P N$ itself and does not require any explicit target maneuver estimation. Guidance Law 2 , based on a first order sliding surface, is designed such that it results in a continuous acceleration law, thereby reducing the chattering problem. While explicitly taking into account the effect of aerodynamic drag, it requires the second derivatives of LOS angle and range, which are not directly measured. An estimation scheme, again based on sliding mode theory, is presented to estimate these quantities. Simulation results clearly demonstrate the superior performance of these schemes.
\end{abstract}

\section{Introduction}

Perhaps, the most widely used guidance law for short range homing missiles is the $\mathrm{PN}$, for reasons of its inherent simplicity and ease of implementation. It is well known that the PN law seeks to null the LOS rate against nonmaneuvering targets, by making the missile heading rate proportional to the LOS rate. Using an on-board seeker, which provides the measurement of the LOS rate, its implementation is straight forward. From a practical point of view, eventhough $P N$ performs reasonably well in a wide range of engagement conditions, its performance degrades sharply in the presence of rapidly maneuvering targets and large off-boresight angle launches. Moreover, the neglected aerodynamic drag affects the missile maneuverability and velocity, resulting in a loss of performance at higher altitudes and in the case of retreating targets.
Recently, Ha et al [1] carried out the performance analysis of the PN law in the presence of target maneuvers and derived conditions which lead to effective intercept. They show that, as long as the initial heading error is small and the navigation ratio is large enough, PN will always result in an intercept with finite missile acceleration. On the other hand, using a differential game theoretic approach, Green et al. [2] consider a coasting pursuer and constant speed target and derive a game optimal guidance law, in closed form. They propose an iterative procedure to estimate some of the variables specified at the final time. Their simulation results depict the capture regions for various launch conditions, indicating a significant improvement over PN. Several other variants of $P N$, and other guidance laws based on optimal control theory have been reported in literature [3-5], all of which claim to give performance improvement over the the basic PN law. A comprehensive survey of guidance laws can be found in Pastrick et al [6] and more recently, in Cloutier et al [7]. The main limitation of these variants, however, is that they require explicit knowledge of target acceleration, missile velocity variation and lateral acceleration, and their performance is critically sensitive to the accuracy of the above information.

Based on the above cursory review, the following general conclusions can be drawn regarding the status of the existing guidance laws. While the simple and easy to implement guidance law, the $\mathrm{PN}$, does not perform equally well in all engagement conditions, the modifications of PN and other guidance laws overcome these limitations at the cost of extra computation and increased complexity, from the implementation angle. Moreover, they suffer from sensitivities associated with respect to the accuracy of the additional information needed. To remove some of these short comings, a nęw class of guidance laws based on Sliding Mode Control (SMC) theory are proposed in [8]. The main advantage of these guidance laws is that they are less sensitive to unmodeled dynamics. More importantly, they are relatively simple to implement and offer robustness against a wide variety 
of target maneuvers.

\section{Planar Engagement Model}

Before proceeding with the derivation of the new guidance laws, the engagement geometry (Fig. 1) which describes the missile-target relative kinematics is presented. For the purpose of simplicity, only a twodimensional engagement in the horizontal plane is considered. The missile is assumed to have a parabolic drag polar and in coasting phase, while the target is assumed to be moving with constant speed. This engagement model based on Reference [2], is represented by the following equations:

$$
\begin{array}{rlrl}
\dot{r} & =v_{t} \cos \left(\gamma_{t}-\theta\right)-v_{m} \cos \left(\gamma_{m}-\theta\right) \\
\dot{\phi} & =\frac{v_{t} \sin \left(\gamma_{t}-\theta\right)-v_{m} \sin \left(\gamma_{m}-\theta\right)}{r} & \\
\dot{\gamma_{m}} & =a_{m} / v_{m} \quad=a_{c} / v_{m} & \left|a_{m}\right| \leq a_{m m a x} \\
\dot{\nu} & =-\left(A v_{m}^{2}+B a_{m}^{2} / v_{m}^{2}\right) & \\
\dot{\gamma_{t}} & =a_{t} / v_{t} & \left|a_{t}\right| \leq \alpha
\end{array}
$$

where

$v_{m}, v_{t}:$ missile and target velocities

$\gamma_{m}, \gamma_{t}:$ the missile and target flight path angles

$r \quad:$ relative range

$\theta$ : LOS angle to the target

$a_{m}, a_{t} \quad$ : missile and target accelerations

$a_{\varepsilon} \quad:$ commanded acceleration of missile

$A, B \quad$ : basic and induced drag related

coefficients of the missile

$a_{\max }, \alpha:$ maximum acceleration limits of the missile and the target

In general, the SMC based system design proreeds in two phases [9]. The first phase entails the construction of a switching surface, so that the system restricted to this surface produces the desired behavior. The next step is to choose a control, which will drive the system trajectories on to the switching surface and constrain them to slide along this surface, for all subsequent time. Robustness can be achieved by choosing the desired surface independent of the external disturbances.

To apply SMC theory to the guidance law design, a suitable form of switching surface, which represents the desired system dynamics, is to be chosen. The selection of the switching surface becomes crucial, because the structure of the guidance law and its robustness properties are both very much dependent on it.

\section{Guidance Law 1}

The hasis for the first guidance law is the invocation of the assumption that missile velocity is constant, which is a consequence of neglecting the aerodynamic drag in this horizontal plane engagement. It is derived by using the switching surface:

$$
S=\dot{\theta}
$$

Nulling the LOS rate provides the motivation for this selection. By a suitable choice of control, if we are able to achieve $\dot{\theta} \equiv 0$, equation (6) together with the assumption that missile velocity $v_{m}$ is greater than $v_{t}$, imply that $\dot{r}<0$, thus guaranteeing interception.

We next design a control law which will guarantee the attractivity of the surface $S=0$ and also the sliding along $S=0$. To carry out this, we construct the following Lyapunov function:

$$
V=\frac{1}{2} S^{2}
$$

which is obviously positive definite. To guarantee the attractiveness of $S=0$, a sufficient condition is to ensure $\dot{V}=\dot{S} S<0$, for $S \neq 0$.

$$
\left.\dot{V}=\frac{\dot{\theta}}{r}\left[-2 \dot{r} \dot{\theta}-a_{m} \cos \left(\gamma_{m}-\theta\right)+a_{t} \cos \left(\gamma_{t}-\theta\right)\right] 8\right)
$$

One particular choice of the control $a_{e}$ which will ensure $V<0$, is

$$
a_{c}=\frac{1}{\cos \left(\gamma_{m}-\theta\right)}[-2 \dot{r} \dot{\theta}+K \dot{\theta}+W \operatorname{sgn}(\dot{\theta})]
$$

where

$$
\operatorname{Sgn}(x)= \begin{cases}1 & \text { if } x>0 \\ 0 & \text { if } x=0 \\ -1 & \text { if } x<0\end{cases}
$$

$W \geq \alpha+\mu, \quad \mu, K>0$

Substituting (9) in (8) we obtain

$$
\begin{aligned}
\dot{V} & =\frac{\dot{\theta}}{r}\left[-K \dot{\theta}-W \operatorname{Sgn}(\dot{\theta})+a_{t} \cos \left(\gamma_{t}-\theta\right)\right] \\
& \leq \frac{\dot{\theta}}{r}[-K \dot{\theta}-\mu \operatorname{Sgn}(\dot{\theta})]<0
\end{aligned}
$$

The choice of $a_{c}$ given by (9) therefore, guarantees attractiveness of the switching surface $S=0$.

Selection of Gains $K, \mu$ : Suppose that $K=-K^{\prime \prime} ;$ and $\mu=-K^{\prime} \rho \dot{r}$. Then, it can be shown that $\dot{\theta}=0$ will be reached at a relative range

$$
r \leq r(o)\left[\rho /(\mid \dot{\theta}(0)+\rho]^{1 / k^{\prime}}\right.
$$

With this choice of $K$ and $\mu$, the guidance law (9) can be written as:

$$
a_{c}=\frac{1}{\cos \left(\gamma_{m}-\theta\right)}[-N \dot{r} \theta+W \operatorname{sgn}(\theta)]
$$

where $W \geq a-h^{\prime} p$ and $N=h^{-1}+2$ 
It is shown in [10] that the additive bias term acts as an estimate of the target acceleration, when the system is in sliding mode steady state. In other words, the guidance law (12) behaves like the APN guidance law in the neighborhood of $\dot{\theta}=0$. This guidance law has been named as the Switched Bias Proportional Navigation (SBPN) and it can be regarded as a PN guidance law with time-varying navigation gain, due to the presence of $\cos \left(\gamma_{m}-\theta\right)$ term and with a switched bias term. This guidance law can also be viewed as a form of the Modified Proportional Navigation (MPN) proposed by Ha et al. [1] for the case of active homing, with the acceleration estimate term in the MPN replaced by the $S g n$ term. However, the main advantage of this guidance law over MPN is that it does not require any explicit target maneuver estimation.

Besides the uncertainty regarding the target acceleration, there exist other unmodeled dynamics like missile velocity variations, neglected seeker and track loop dynamics, etc. A way of coping with these uncertainties is to determine their bounds and include them in the uncertainty bound parameter $\alpha$, which in the above derivation represented the target acceleration bound alone. In general, it is often difficult to find exact bounds for each of these uncertainties. While a conservative bound may require excessive control effort in the reaching phase, an underestimated bound may violate the reaching condition and in consequence can lead to loss of stability.

In order to resolve this problem, $\alpha$ is replaced with $\alpha$ in (12) where $\hat{\alpha}(t)$ is given by

$$
\hat{\alpha}(t)=\alpha_{0}+\alpha_{1} \int_{0}^{t}|r \dot{\theta}| d \zeta
$$

where $\alpha_{0}$ and $\alpha_{1}$ are some positive numbers.

A detailed derivation which leads to the above can be found in [10]. The main advantage of the above bound is that instead of using conservative estimates of $\alpha$, which result in a large control effort, one can start with a less conservative estimate $\alpha_{0}$, and can still maintain the attractivity of the switching surface, even in the case of an underestimated initial uncertainty bound, represented by $\alpha_{o}$.

With these modifications, the SBPN law assumes the final form :

$$
\begin{aligned}
a_{c}=-\frac{N}{\cos \left(\gamma_{m}-\theta\right)} \dot{r} \dot{\theta}+W S g n(\dot{\theta}) \\
W \geq \alpha-K^{\prime} \rho \dot{r} \\
\alpha=\alpha_{0}+\alpha_{1} \int_{0}^{t}|r \dot{\theta}| d \zeta
\end{aligned}
$$

where $K^{\prime \prime}, \rho, \alpha_{0}$ and $\alpha_{1}$ are some positive real numbers.

The simulation results in section $V$, provide some insights into the performance of this guidance law.

\section{Guidance Law 2}

In the previous section, the selection of $S=\dot{\theta}$ as a switching surface has resulted in a guidance law which is a variant of the PN law. One drawback with this specific choice of switching surface is that in the case of highly maneuvering targets and large initial heading errors, equation (14) requires large gains for the $\dot{\theta}$ trajectories to reach and slide along the switching surface. Another limitation is that like the PN, the SBPN also does not directly take into account the effect of aerodynamic drag on the missile speed variation. These can be overcome by considering the alternative switching surface:

$$
S=r \ddot{\theta}-N_{s} \dot{r} \dot{\theta}
$$

The motivation for the selection of the above switching surface can be explained as follows. Suppose that we are able to design a guidance law which will ensure the reaching condition and hence the system trajectories slide along the switching surface $S$, in the sliding mode steady state. Then $S \equiv 0$ implies that

$$
\dot{\theta}=\dot{\theta}_{0}\left(r / r_{o}\right)^{N_{s}}
$$

and if the guidance law can ensure $\dot{r}<0$ during the reaching phase, then $\dot{\theta}$ will be driven to zero while closing in on. range. In other words, this choice of switching surface allows for a gradual decay of $\dot{\theta}$, rather than an instantaneous nulling. The first order switching surface partially compensates for the autopilot dynamics and the system delays, which are not explicitly modeled.

The guidance law, which guarantees the sliding condition, can be derived along the same lines as in the previous section. In particular, with the choice of equation (7) as the Lyapunov function, the attractivity of the $\dot{\theta}$ trajectories to the switching surface $S=0$ can be guaranteed by maintaining

$$
\dot{S}<-P S-D \dot{\operatorname{Sgn}(S)}
$$

where $P$ and $D$ are some positive numbers. It can be shown that the trajectory of $S$ will reach the sliding surface $S=0$ in a time $t_{s}$ less than $1 / P \ln [(|S(0)|+$ $D) / D$ ], where $S(0)$ is the initial value of $S$ at time $t=0$. Consider the following choice for $\dot{a}_{c}$ :

$$
\begin{aligned}
& \begin{aligned}
\dot{a}_{c}= & -\left(\left(\left(N_{s}+2\right) \dot{r}-P r\right) \ddot{\theta}-\left(\left(N_{s}+3\right) \ddot{r}-P N, \dot{r}\right) \dot{\theta}\right. \\
+ & \left.(D+\mu) \operatorname{Sgn}(S)+a_{m}^{2} / v_{m} \sin \left(\gamma_{m}-\theta\right)\right) / \\
& \left(\cos \left(\gamma_{m}-\theta\right)-2 B a_{m} / v_{m}^{2} \sin \left(\gamma_{m}-\theta\right)\right)
\end{aligned} \\
& \text { where } \mu>\dot{a}_{t \max }+\alpha^{2} / v_{t}+|f|_{\max } \text { and } \\
& \qquad \begin{aligned}
f= & -2 A v_{m} \dot{v}_{m} \sin \left(\gamma_{m}-\theta\right)-\dot{v}_{m} a_{m} \cos \left(\gamma_{m}-\theta\right) / v_{m} \\
+ & 2 B a_{m}^{2} / v_{m}^{3} \ddot{v}_{m} \sin \left(\gamma_{m}-\theta\right)
\end{aligned}
\end{aligned}
$$


Here the term $f$ represents the effects of the missile velocity variation due to drag. By differentiating $S$ with respect to time, substituting (18) for $\dot{a}_{c}$ and writing in terms of $\ddot{\theta}$ and $\ddot{R}$ we obtain :

$$
\begin{aligned}
\dot{S} & =-P S-\left(D+\mu i \operatorname{Sgn}(S)+\dot{a}_{t} \cos \left(\gamma_{t}-\theta\right)\right. \\
& -a_{t}^{2} / v_{t} \sin \left(\gamma_{t}-\theta\right)+f
\end{aligned}
$$

With the value of $\mu$ selected as above, it can be shown that (17) is satisfied.

The equation (18) can lie regarded as a form of dynamic feedback guidance law, where the commanded acceleration rate becomes the control variable. The name $S$ Witched Acceleration Rate (SWAR) guidance law is chosen for (18) to signify that the guidance command is the acceleration rate and an acceleration dependent ign() term is also present in the expression.

It can be observed that the magnitude of the missile acceleration rate is a function of the induced drag coefficient, thereby requiring the missile to accelerate slowly in the presence of large induced drag. The first two terms on the right hand side of (18) are intended to null the LOS rate. The $S g n$ term is added to compensate for the target acceleration and jerk, while the last term takes into account the missile velocity variations. Note that under the assumptions of constant speed for the missile, a non maneuvering target, and a small off-boresight angle launch, the above guidance law can be shown to reduce to the conventional PN. Alternatively, if we drop the aerodynamic drag and the missile acceleration terms from equation (18), it can be regarded as a special form of the SBPN, with the switching term in the latter being replaced by an integrated signum function of the LOS rate and its derivative.

From the structure of the guidance law, it is clear that it requires knowledge of the acceleration terms $\ddot{R}$ and $\ddot{\theta}$. Since these quantities in turn directly depend on the target acceleration and other system dynamics like the autopilot delays, it implies that these effects are explicitly taken into account when one is using $a_{c}$, as given by (18). Also, the dynamic nature of this guidance law makes the missile acceleration at least first order continuous, thereby reducing the effect of chattering.

However, a basic limitation of this approach is that $\ddot{R}$ and $\ddot{\theta}$ are to be estimated, which increases the complexity associated with the implementation of this guidance law. In the next section, an estimation scheme, again based on SMC theory is proposed to provide estimates of these quantities.

\section{Sliding Mode Estimator}

'The engagement geometry, which describes the missile and target relative states, is inherently nonlinear. Many of the conventional schemes for the estimation of the missile-target relative states are based on the Extended Kalman Filter (EKF). The robustness properties of these schemes are not well established. The sliding estimators, based on SMC theory, are inherently nonlinear and they offer robustness with respect to unmodeled dynamics. In this section, an SMC based estimator is designed to estimate the $\ddot{R}$ and $\ddot{\theta}$, and its performance investigated.

The polar coordinate frame is selected for the implementation of the estimator because, as stressed by Cloutier et al [7], the polar implementation produces a simpler and more accurate estimator than its cartesian counterpart. Moreover, the application of SMC theory is relatively less complicated in the case of a linear measurement model. In this coordinate frame, the estimator state and measurement models can be represented as:

$$
\begin{aligned}
\dot{X} & =f\left(X, a_{t}, \dot{a}_{t}\right) \\
y & =H X+w
\end{aligned}
$$

where $X=\left[\begin{array}{lllllll}r & \dot{r} & \ddot{r} & \dot{\theta} & \ddot{\theta} & a_{m} & \dot{a}_{m}\end{array}\right]^{\prime}$ and

$$
f=\left[\begin{array}{c}
\dot{r} \\
\ddot{r} \\
\Phi_{r} \\
\ddot{\theta} \\
\Phi_{\theta} \\
\dot{a}_{m} . \\
-\zeta \omega_{n} \dot{a}_{m}-\omega_{n}^{2}\left(a_{c}-a_{m}\right)
\end{array}\right]
$$

and

$$
H=\left[\begin{array}{lllllll}
1 & 0 & 0 & 0 & 0 & 0 & 0 \\
0 & 1 & 0 & 0 & 0 & 0 & 0 \\
0 & 0 & 0 & 1 & 0 & 0 & 0 \\
0 & 0 & 0 & 0 & 0 & 1 & 0
\end{array}\right]
$$

$$
\begin{aligned}
\Phi_{r} & =3 \dot{\theta}(\dot{r} \dot{\theta}+r \ddot{\theta}) \\
& +\dot{a}_{m}\left(\sin \left(\gamma_{m}-\theta\right)+2 B a_{m} \cos \left(\gamma_{m}-\theta\right) / v_{m}^{2}\right) \\
& -\dot{a}_{t} \sin \left(\gamma_{t}-\theta\right)-a_{t}^{2} / v_{t} \cos \left(\gamma_{t}-\theta\right)+f_{\nu} \\
\Phi_{\theta} & =\left(-3 \ddot{r} \dot{\theta}-3 \dot{r} \ddot{\theta}+r(\dot{\theta})^{3}\right. \\
& -\dot{a}_{m}\left(\cos \left(\gamma_{m}-\theta\right)-2 B a_{m} \sin \left(\gamma_{m}-\theta\right) / v_{2}^{m}\right) \\
& +a_{m}^{2} / v_{m} \sin \left(\gamma_{m}-\theta\right) \\
& \left.+\dot{a}_{t} \cos \left(\gamma_{t}-\theta\right)-a_{t}^{2} / v_{t} \sin \left(\gamma_{t}-\theta\right)\right) / r+g_{\nu}(2)
\end{aligned}
$$

where $w$ is the additive measurement noise vector and $\zeta$ and $\omega_{n}$ are the missile autopilot damping ratio and natural frequency respectively. The $f_{\nu}$ and $g_{\nu}$ terms in the above represent the contribution of aerodynamic drag. It is assumed here that the missile is equipped with both a passive gimballed seeker and an active Doppler seeker. The measurement of LOS rate $\dot{\theta}$, is available from the passive seeker and the $r, \dot{r}, \theta$ are available from the active seeker. Further, it is assumed that the missile 
is equipped with an accelerometer to measure the missile acceleration, represented by $a_{m}$. The measurement noises are assumed to be gaussian distributed, with zero mean and variances, as given in the next section on simulation studies.

Using the state space representation developed in the previous section, a nonlinear maneuver estimator has been developed. With the estimation errors as the switching surfaces, application of the SMC estimation theory, as described in Slotine et al [11], results in the following estimator structure.

$$
\dot{\dot{X}}=f(\hat{X})+K_{I}(\tilde{y})+K_{s} \operatorname{Sgn}(\tilde{y})
$$

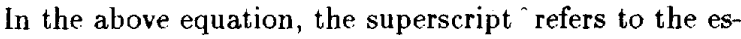
timated states and refers to the estimation error in the measured states, i.e. $\tilde{y}=y-\hat{y} . K_{I}$ and $K_{s}$ are the gain matrices which are to be determined.

It is shown in [11] that, though the effect of adding a signum term to the estimator equations is to increase the effective bandwidth of the filter, the SMC based estimator possesses the remarkable property of increasing the effective bandwidth without potentially increasing the noise sensitivity. This is particularly true at high data rates and low noise intensity, as brought out by simulation results presented in the next section.

The above Sliding Mode state estimator is in the standard Luenberger form, except for the additional nonlinear switching terms. It is these terms which impart robustness to the estimator. The main difference between the Sliding Mode estimator and the EKF is that the former explicitly takes into account the effect of disturbances and compensates for them, while in the latter, the disturbances are accommodated in the form of process noise. This is the main reason for their superior performance over the conventional EKF based schemes.

Since the linear gains $K_{I}$ do not influence the sliding condition, they can be selected rather independently to optimize the performance of the estimator. For example, they can be taken as the corresponding EKF gains. The nonlinear gain matrix $K_{s}$, is however, to be chosen large enough to satisfy the sliding condition [11]. With this selection, the estimator assumes the form:

$$
\begin{aligned}
\dot{\hat{X}} & =f(\hat{X})+K_{I}(\tilde{y})+K_{s} \operatorname{Sgn}(\tilde{y}) \\
\dot{P} & =F(\hat{x}) P+P F(\hat{x})^{T}-P H^{T} R^{-1} H P+Q(27) \\
K_{I} & =P H^{T} R^{-1}
\end{aligned}
$$

where $\mathrm{P}$ is the covariance of the state estimation error and $F$ is the Jacobian of $f$ with respect to the estimated state $\hat{X}$.

Once the gains are chosen to satisfy the requirement of the sliding condition, the sliding will be reached in a finite time and the approximate error dynamics on the sliding patch are governed by

$$
\tilde{y}=0
$$

The performance of the above filter has been investigated in the in the following section.

\section{Simulation Results}

In this section, the performance of the two SMC based guidance and estimation schemes are evaluated in the presence of maneuvering targets and other representative engagement conditions. For the purpose of comparison, the following versions of PN and APN are employed in the simulation studies.

$$
\begin{array}{r}
a_{c p n}=-\left(N^{\prime} / \cos \left(\gamma_{m}-\theta\right)\right) \dot{R} \dot{\theta} \\
a_{c a p n}=-\left(N^{\prime} / \cos \left(\gamma_{m}-\theta\right)\right) \dot{R} \dot{\theta}+a_{t} \cos \left(\gamma_{t}-\theta\right)
\end{array}
$$

Where, $a_{c p n}$ and $a_{c a p n}$ denote the commanded accelerations with PN and APN respectively.

Before proceeding with the simulation studies, the structure of the guidance law (14) is slightly modified, mainly from implementation considerations. One of the underlying assumptions in the design of these guidance laws has been that the missile acceleration can be instantaneously switched from one value to another, at will. In practice, however because of various -system nonidealities, switching of the control signal at a very fast rate will result in chattering of the control signal, which in turn may excite the unmodeled high frequency dynamics. The chattering can be eliminated by replacing the discontinuous $\operatorname{sgn}()$ with a continuous approximation, like the high gain saturation function. In the present study, an approximation of the form $(x /(|x|+\delta)$ where $\delta>0$ is a small real number, has been used instead of $\operatorname{Sgn}(x)$. Though, such a problem does not arise with SWAR, the $\operatorname{Sgn}()$ term in (18) is also modified similarly, mainly to make the acceleration profiles smoother in the presence of noise.

Engagement scenarios : Two different engagement situations are considered for the purpose of simulation. In both the cases, the target is assumed to pull maximum acceleration throughout the engagement. These two engagements are identical in nature, except for the initial range considered. In the first case, the initial range to the target is assumed to be $3 R_{\text {ref }}$ and in the latter, it is $5 R_{\text {ref }}$ where $R_{\text {ref }}=1515.15 \mathrm{mtrs}$. The short range engagement brings out the effectiveness of the guidance laws in the presence of maneuvering targets, whereas the long range enagement demonstrates the impact of the presence of aerodynamic drag. Table 1 gives the simulation data used. A final separation of less than ten meters between the missile and the target is deemed to be interception, in the cases studied.

Deterministic Studies : In this case, it is assumed that all quantities required for the implementation of the guidance laws are available without any measurement errors. In particular, the exact value of target 
acceleration needed for the APN and the $\ddot{R}$ and $\ddot{\theta}$ required for the SWAR, are assumed to be available. The cases that deal with the noisy measurements and where the above quantities are to be estimated, are presented in the sub section on stochastic studies. To impart realism to the studies, second order autopilot and first order seeker dynamics are introduced.

Figures 2 and 3 give the commanded acceleration profiles for the two engagement situations. While the PN requires excessive acceleration towards the end, the acceleration profiles of both the SBPN and SWAR show trends similar to the APN. Note that the initial value of the commanded acceleration required in SWAR has been set to the corresponding $\mathrm{PN}$ value.

Table 2 gives the final velocities and flight times for the two engagements studied. In the long range case, the PN fails to intercept the target (miss distance $317 \mathrm{mtr} \mathrm{r}^{\mathrm{s}}$ ). The APN has slightly higher values of missile velocity, while those of SBPN and SWAR are almost the same.

The LOS rate profiles, given in Figures 4 and 5 indicate that, while SBPN, SWAR and APN show a similar trend, those of PN exhibit a completely different behavior.

From the switching surface time histories for SWAR, shown in Fig. 6, it can be observed that the system is drawn quickly to the sliding mode and it remains on it.

The contribution of the different terms on the right hand side of (18) to the commanded acceleration rate are shown in Fig. 7 . The notation, 'Sgn' and ' $a_{m}$ ', used in Fig. 7 indicates the contributions of the modified $5 g n$ and the $a_{m}$ terms in (18) to the commanded acceleration rate. From this figure, it may be inferred that, the contribution of the missile acceleration term to the commanded acceleration steadily decreases and the LOS rate terms remain the major contributors. Perhaps, this accounts for the marginal performance improvement seen of SWAR over SBPN, eventhough, the latter explicitly takes into account the effect of aerodynamic drag

Stochastic Studies The performance of the guidance laws and the sliding mode estimator scheme proposed has been investigated for the short range engagement case, with the simulation data given in Table 1. From the $\ddot{R}$ and $\ddot{\theta}$ estimate profiles, shown in Figures 8 and 9 , it can be observed that the $S g n$ terms in the SMC estimator indeed give rise to improved estimates of these quantities. The noisy nature of the estimates can be attributed to the rather non-optimal selection of the parameters involved. The performance of both the EKF and the SMC based estimators can be further improved by proper tuning of their respective parameters. Nonetheless, these results are indicative of the performance of the SMC based estimation scheme.

Figure 10 shows the commanded acceleration profiles for the PN, SBPN and SWAR schemes. The APN is omitted from this comparison because of the nonavail- ability of the target acceleration estimate. From this figure, it can be observed that while there is general degradation in the performance of all the three schemes, the SWAR acceleration profiles are relatively less noisy and also require less acceleration. These results clearly provide a pointer to the robustness of the SMC: based guidance schemes over the $\mathrm{PN}$, in the presence of maneuvering targets and missile velocity reduction due to aerodynamic drag.

\section{Conclusions}

Two new guidance laws for short range homing missiles based on Sliding Mode Control theory are presented and their performance is investigated in the presence of target maneuvers and aerodynamic drag. The simulation results presented clearly bring out the superior performance features of these guidance laws over PN.

\section{References}

[1] Ha, I. J, Hur. J. S, Ko. M. S and Song, T. L, " Performance Analysis of PNG Laws for Randomly Maneuvering Targets", IEEE. Trans. on Aerospace and Electronic Systems, Vol. AES- 26, No. 5, 1990, $713-721$.

[2] Green, A., Shinar, J. and Guelman, M., "('ame optimal Guidance Law Synthesis for Short Range Missiles", Journal Of Guidance, Control and Dynamics, Vol. 15, No. 1, 1992, pp. $191-197$.

[3] Speyer, J. L., "An Adaptive Terminal Guidance Scheme Based on Exponential Cost Criterion with Application to Homing Missiles", IEEE Trans. Automatic Control, Vol. AC - 21, June 1976, pp. $371-375$.

[4] Riggs, T. L., "Linear Optimal Guidance Laws for Short Range Air-to-Air Missiles", Proc. of National Acrospace and Electronic Systems Conference, 1979, pp 757-764.

[5] Guelman, M. and Shinar, J., "Optimal Guidance Law in the Plane", Journal of Guidance, Dynamics and Control, Vol. 7, No. 4, July-August 1984, pp 471-476.

[6] Pastrick, H. J., Setlzer, S. and Warren, M. E., "Guidance Laws for Short Range Homing Missile", Journal of Guidance, Control and Dynamics, Vol.4, No. 2, March April 1981, pp. $98-108$.

[7] Cloutier, J. R., Evers, J. H. and Feeley, J. J., "Assessment of Air to Air Missile and Guidance Technology",IEEE Control Systems Magazine, Vol. 9, October 1989, pp. 27-34.

[8] Ravindra Babu, K., "VSC-Based Schemes for Homing Guidance and Maneuvering Target Estimation", Ph.D thesis under preparation, Dept. of Computer Science and Automation, Indian Institute of Sicience.

[9] Utkin, V. I., "Variable Structure Systems with Sliding Modes", IEEE. Transactions on Automatic Control, Vol. A('-22. No. 2, 1977 , pp. 212- 222 
[10] Rarindra Habu. K. Sarma. I.( and Swamly $K$ $\therefore$. "Switched Bias I'roprersional Navigation for Homiug (illidance dgainst llighly Manenvering Titgets", arcepted for publication in Jonrual of Ciudance, Control and llynamess.

[11] Slotine. J.J E. Hedrick. J.h and Misawa, E.A. Nonlinear State Observation lising Sliding Ob-

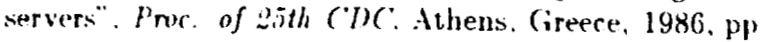
$32: 3-3) 29$

Table 1 Simulation Data

\begin{tabular}{llll}
\hline \hline$r_{m}$ & $800 m / s$ & $r_{t}$ & $300 m / s$ \\
$\theta$ & $0^{\circ}$ & $\gamma$ & $105^{\circ}$ \\
$i m$ & $40^{\prime \prime}$ & $a_{n}$ & 20.0 \\
$\gamma$ & 0.0007 & $N^{\prime}$ & 4 \\
$\prime^{\prime}$ & 0.02 & $D$ & 50.0 \\
$\Gamma^{\prime}$ & 0.0 & $N s$ & 2.0 \\
$N^{\prime}$ & 1.0 & $A$ & 132.5 \\
$B^{\prime}$ & 606.06 & $R_{\text {ref }}$ & 1515.15
\end{tabular}

Measurement Variance

kange

$10\left(m m^{2}\right.$

Ratlge Rate

$100 m^{2} / s^{2}$

LOS Rall.

$25 \times 10^{-i} \operatorname{rad}^{2} / \mathrm{s}^{2}$

Table 2 Final velucities and Flight Times

\begin{tabular}{|c|c|c|c|c|}
\hline \multirow{2}{*}{$\begin{array}{c}\text { Guidlimes } \\
\text { Law }\end{array}$} & \multicolumn{2}{|c|}{$l i=3 h_{r+1}^{\prime}$} & \multicolumn{2}{|c|}{$k=5 h_{r e f}$} \\
\hline & veloculy & linne & velocity & lime \\
\hline & $m 1 / \mathrm{s}$ & seer & $n 1 / s$ & $\sec$ \\
\hline$A P N$ & ה73.183 & 5.30 & .446 .32 & 9.931 \\
\hline$P$ & 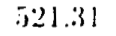 & 5.35 & 315.49 & 11.5 \\
\hline SBPN & 307.18 & 5.51 & אים.8. & 10.06 \\
\hline SWLAR & 5640.22 & $5.3 \% 2$ & 412.7 & 10.103 \\
\hline
\end{tabular}

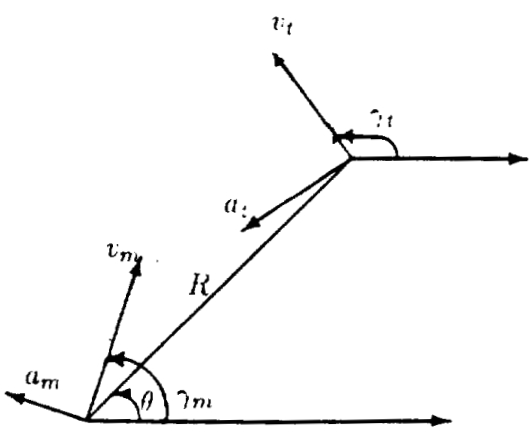

Fir. 1 Engagement Geometry

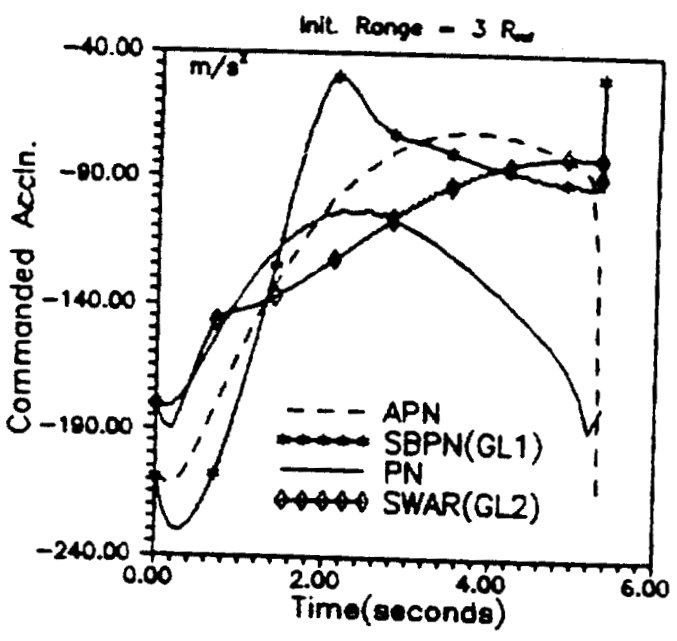

Fig. 2 Commonded Acceleration Profiles

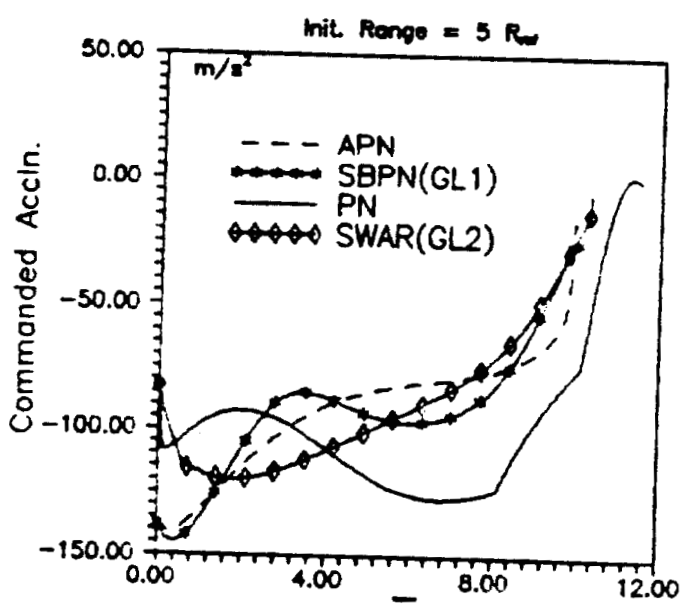

Fig. 3 Commanded Acceleration Profiles

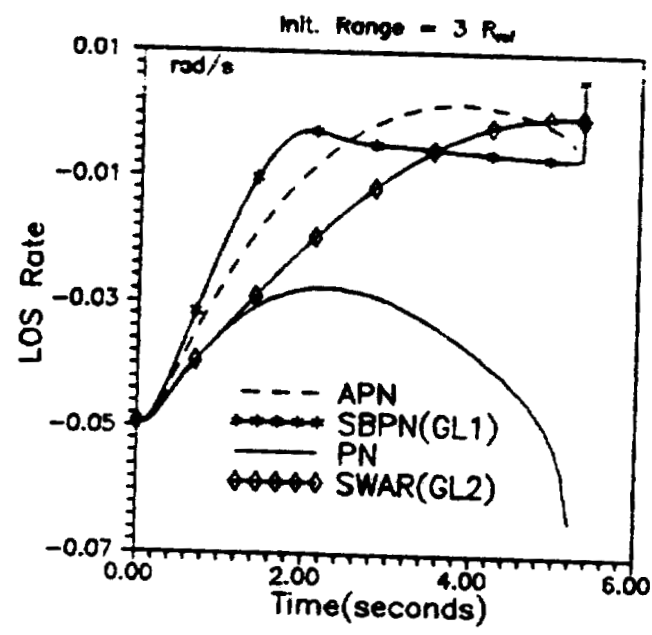

Fig. 4 LOS Rote Profiles 


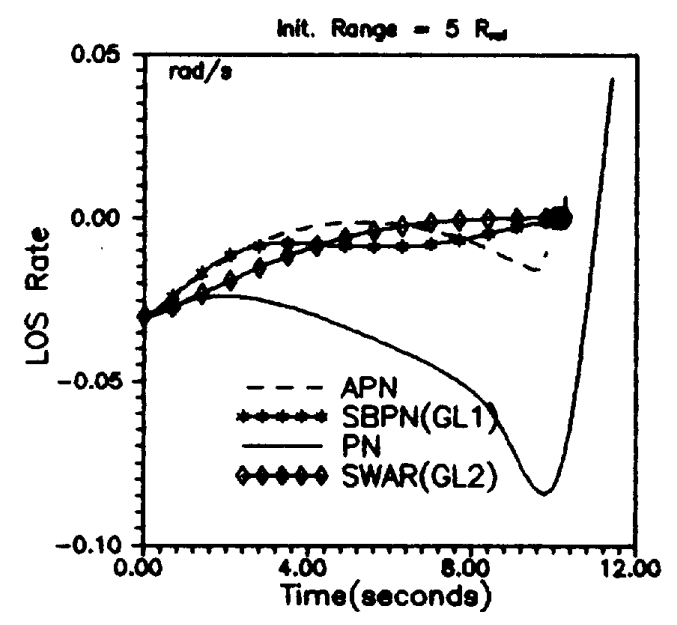

Fig. 5 LOS Rate Profiles

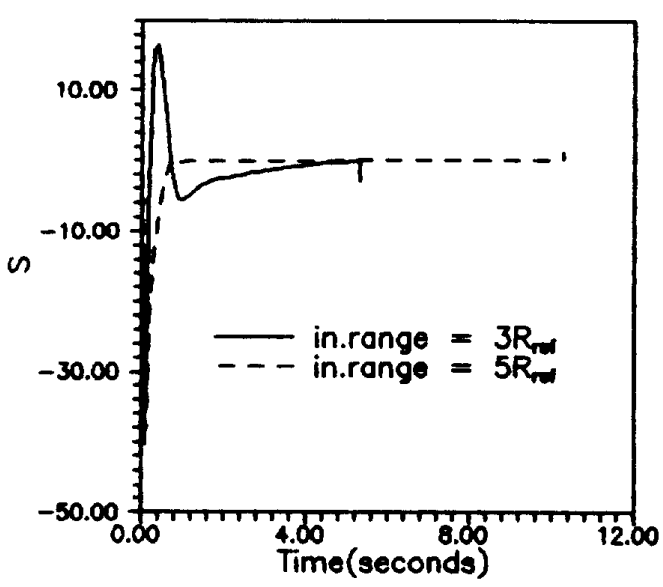

Fig. 6 Switching Surface for SWAR (GL2)

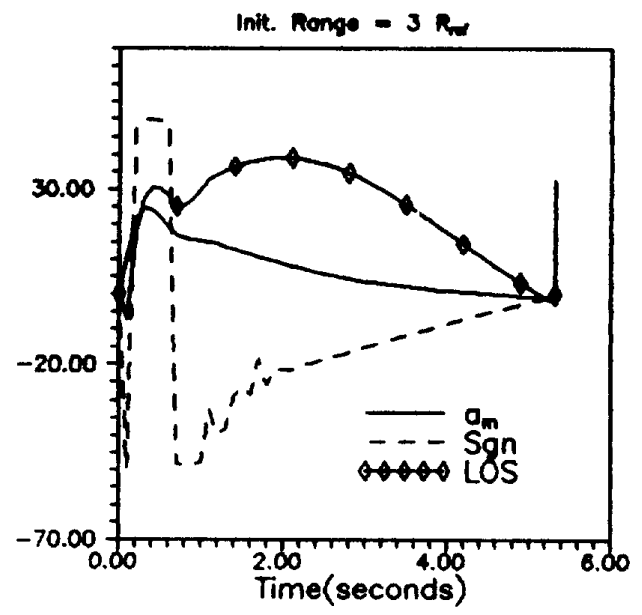

Fig. 7 Contribution of different terms in SWAR(GL2)

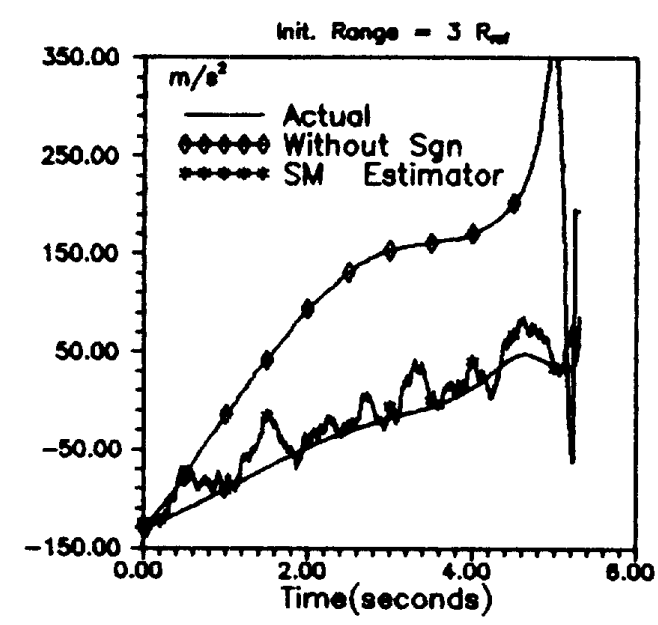

Fig. $8 \ddot{R}$ Estimate Profiles

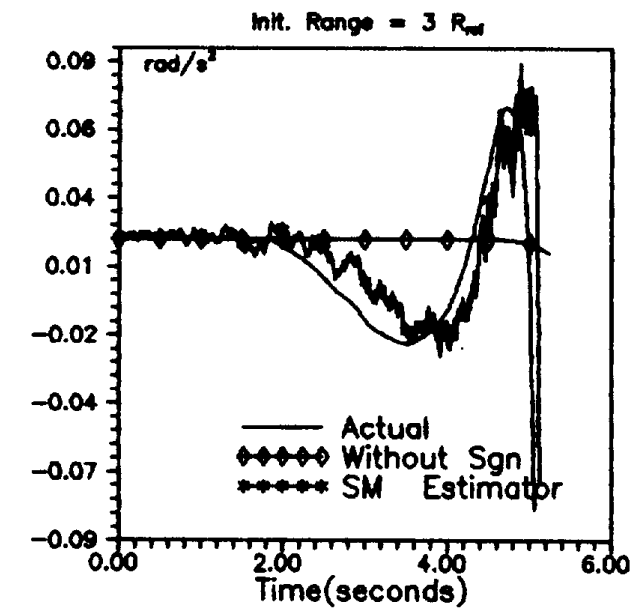

Fig. $9 \ddot{\theta}$ Estimote Profiles

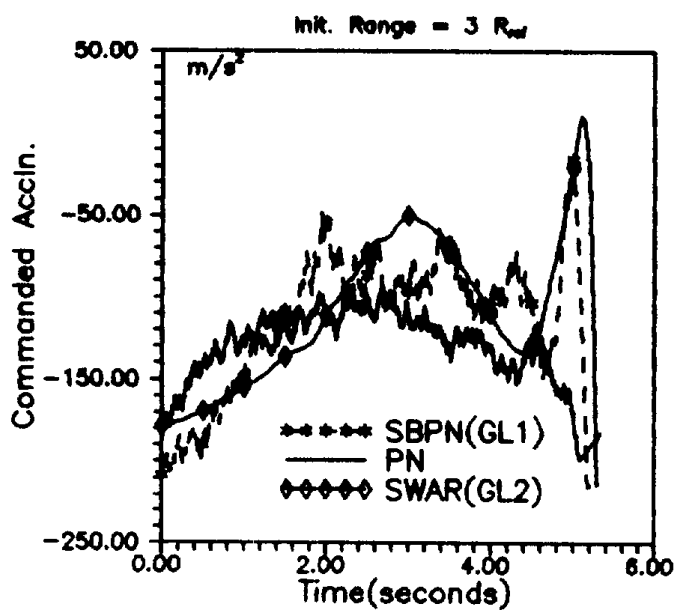

Fig. 10 Commanded Acceleration Profiles (stochastic cose) 